\title{
Small molecules modulating tumor-stromal cell interactions: new candidates for anti-tumor drugs
}

\author{
Manabu Kawada \\ The tumor microenvironment comprises tumor cells surrounding normal cells and the extracellular matrix. The surrounding \\ normal cells have critical roles in the regulation of growth and metastasis of cancers, including the maintenance of cancer stem \\ cells and the formation of cancer metastatic niches. Recent anti-tumor strategies targeting the tumor microenvironment have \\ included inhibition of angiogenesis and the augmentation of immune reactions. However, in this review, we will focus on stromal \\ cells (fibroblast-like cells), a common constituent of the tumor microenvironment. Since stromal cells regulate the growth of \\ cancer cells positively and negatively through adhesion and secreted factors, anti-tumor strategies should consider modulating \\ tumor-stromal cell interactions through use of small molecules.
}

The Journal of Antibiotics (2016) 69, 411-414; doi:10.1038/ja.2016.37; published online 23 March 2016

\section{INTRODUCTION}

Cancer chemotherapy results have been dramatically improved in recent years by the development of molecularly targeted drugs. However, the incidence of drug resistance because of the instability of the cancer cell genome is a serious problem. In contrast, stromal cells are normal and their genomes are relatively stable compared with those of cancer cells. Thus, anti-tumor drugs targeting stromal cells could avoid the development of drug resistance. Furthermore, such drugs would have mechanisms of action differing from traditional anti-tumor drugs. Thus, the use of both types in combined treatment might be particularly effective.

\section{STROMAL CELLS REGULATE THE GROWTH OF CANCER CELLS}

The tumor microenvironment is composed of cancer cells and stroma such as extracellular matrix and various types of cells. The latter includes macrophages, endothelial cells, immune cells and fibroblast-like cells (here we call them stromal cells). This review will focus on stromal cells. The stromal cells are also termed cancer-associated fibroblasts, and they have critical roles in the maintenance of cancer stem cells ${ }^{1}$ and the formation of cancer metastatic niches. ${ }^{2}$ Stromal cells maintain the growth and characteristics of normal epithelial cells and epithelial derived cancer cells. Host processes like wound healing act on cancer cells in a favorable way. Stromal cells secrete growth factors and proteases that assist the growth and metastasis of cancer cells.

As shown in Figure 1a, two gastric cancer cell lines formed tumors in nude mice when inoculated subcutaneously. However, the co-inoculation of gastric stromal cells resulted in surprising outcomes. Although the growth of one of the tumors was apparently increased by the gastric stromal cells, that of the other tumor was significantly decreased in the presence of the gastric stromal cells. Thus, these results clearly showed that stromal cells can regulate tumor growth positively and negatively through secreted factors and direct cell-cell adhesion in tumor-stromal cell interactions. These results suggest that it might be possible to use stromal cells' negative regulators to develop new anti-tumor strategies. Toward that end, we have studied tumor-stromal cell interactions.

\section{SMALL MOLECULES MODULATING TUMOR-STROMAL CELL INTERACTIONS}

As stromal cells regulate the growth of cancer cells through secreted factors and direct cell-cell adhesion, cancer cells also regulate the characteristics of stromal cells. Growth factors such as HGF and TGF- $\beta$ are reported to participate in tumor-stromal cell interactions; however, the precise mechanisms remain to be elucidated because of the diversity of factors among organs and tissues. ${ }^{3}$ To reveal critical factors involved in tumor-stromal cell interactions and to use those interactions in anti-tumor strategies, we have been analyzing various small molecules that can modulate the interactions. Furthermore, by analysis of the mechanisms of actions of the small molecules, we have been elucidating some factors that have critical roles in the interactions.

For the screening of active small molecules we established a co-culture system, in which cancer cells are co-cultured with stromal cells (Figure 1b). In this way, we have screened compounds that can inhibit the growth of cancer cells in co-culture with stromal cells but cannot affect the growth of cancer cells cultured alone or that of 

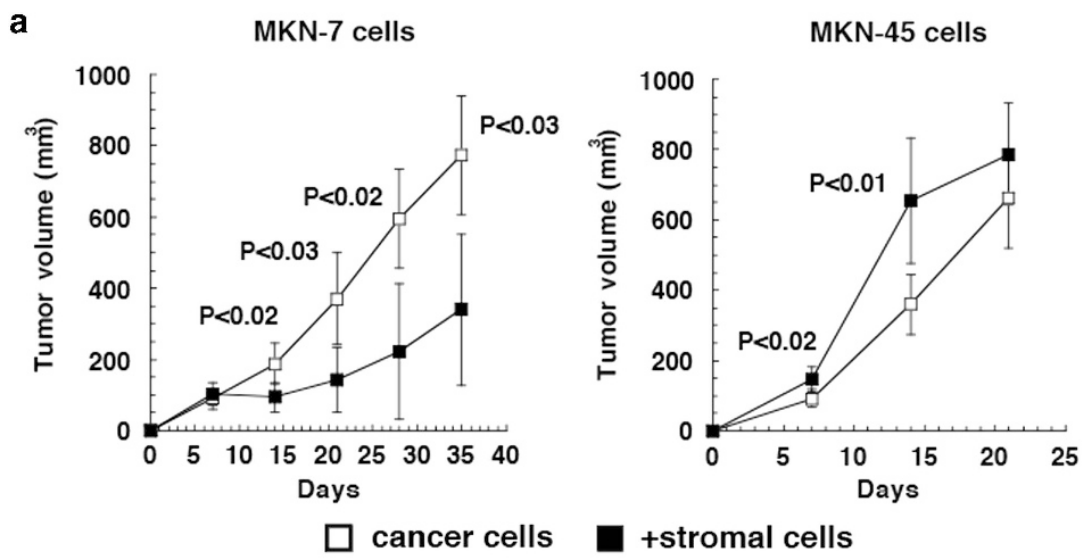

b

Coculture system

Activity of a hit compound

Stromal cells
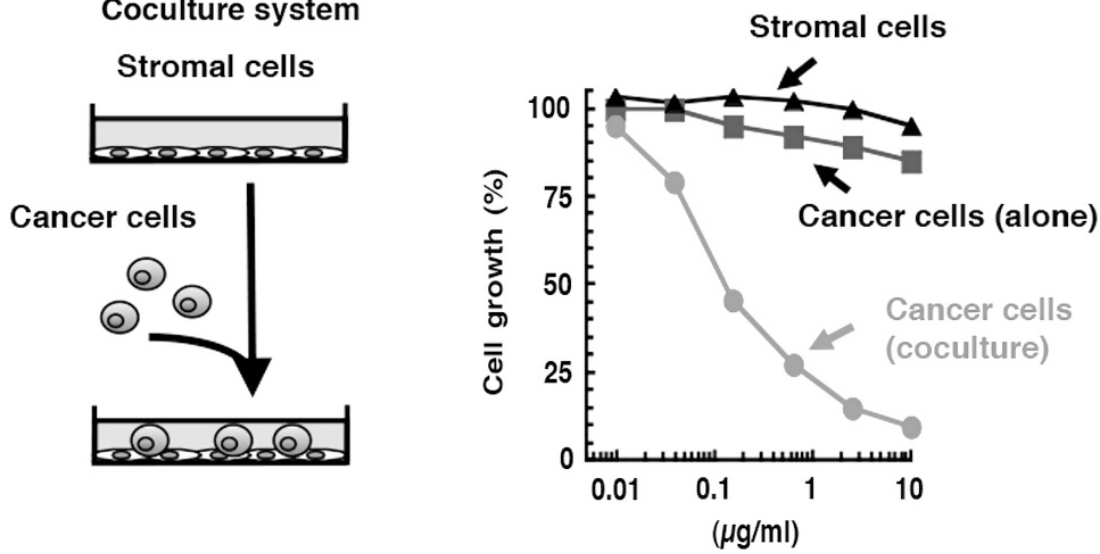

Figure 1 Tumor-stromal cell interactions. (a) Human gastric cancer cells were subcutaneously injected into nude mice with or without gastric stromal cells. Stromal cells could decrease (left) or increase (right) the tumor growth in vivo. (b) For co-culture experiments, cultures are initiated with stromal cells after which cancer cells are directly inoculated onto the layer of stromal cells. Active compounds show selective growth-inhibitory activity against cancer cells co-cultured with stromal cells, but not against cancer cells or stromal cells cultured alone.

stromal cells (Figure 1b). We introduce small molecules showing such activity in the following sections.

\section{ANALYSIS OF TUMOR-STROMAL CELL INTERACTIONS USING COMMERCIALLY AVAILABLE INHIBITORS}

To elucidate factors that are active in tumor-stromal cell interactions, we used various commercially available inhibitors. Using our co-culture system and this screening process, we obtained many new findings.

\section{IGF-IR inhibitor}

When we examined the effects of various small molecule inhibitors in a co-culture system of human prostate cancer cells and prostate stromal cells, we found that AG1024 (Figure 2), an inhibitor of IGF-IR tyrosine kinase, affected prostate tumor-stromal cell interactions. The growth of prostate cancer is increased by co-culture with prostate stromal cells. ${ }^{4}$ Studies of its mechanism of action revealed that prostate stromal cells secrete IGF-I that is used as a growth factor by the tumor cells. ${ }^{4}$ In short, AG1024 was found to inhibit the action of IGF-IR, a receptor for IGF-I, in prostate cancer cells and thus suppressed the growth of prostate cancer in the co-culture with stromal cells. The production of IGF-I was detected in human prostate cancer tissues, indicating that the local production of IGF-I in the prostate is a feasible molecular target for cancer treatment.

\section{MEK inhibitor I}

We found that MEK inhibitor I was a modulator of tumor-stromal cell interactions when we screened a co-culture of human gastric cancer cells and gastric stromal cells (Figure 2). However, U0126, another well-known MEK inhibitor, did not show the same activity suggesting that the action of MEK inhibitor I was irrelevant to MEK. Our mechanistic study revealed that MEK inhibitor I bound to RPL-18A, a 60 S ribosomal protein, and inhibited IL-6 production from gastric stromal cells. ${ }^{5}$ On the other hand, we found that gastric cancer cells secreted TNF $\alpha$ and PGE2 and these mediators stimulated gastric stromal cells to secrete IL-6. ${ }^{5}$ Thus, gastric cancer cells grow using this source of IL-6 (Figure 3a). While IL-6 activates Stat 3 in gastric cancer cells, we detected the activation of Stat3 in about half of human gastric cancer tissues. ${ }^{5}$ Furthermore, MEK inhibitor I was found to increase the secretion of growth-inhibitory factors against gastric cancer cells from gastric stromal cells. We then tried to identify the factor in the conditioned medium from gastric stromal cells. As a result, we found that GAPDH, a well-known house keeping protein, is secreted from gastric stromal cells and inhibits protein synthesis, resulting in growth suppression by binding to E-cadherin on cancer cells (Figure 3a). ${ }^{5}$ Although it is reported that GAPDH is secreted extracellularly and affects cell morphology, ${ }^{6}$ we found that it acts as a growth-inhibitory factor. Furthermore, GAPDH is secreted by various stromal cells in 
<smiles>CC(C)(C)Cc1cc(Br)c(O)c(C(C)(C)C)c1</smiles>

AG1024<smiles>CC(=O)C(C)(C)C(C)C(C)CCCCCCC1CNCO1</smiles>

Phthoxazolin A<smiles>CC(=CCCC=CCCc1cnco1)C(C)C(C)C(C)O</smiles>

Inthomycin B

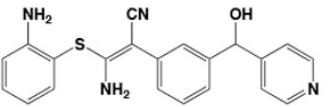

MEK inhibitor I

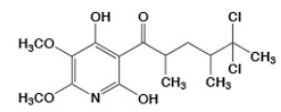

NBRI23477 A<smiles>CCC(C)CC1CN=C(OC)C(OC)C(O)C1=O</smiles>

NBRI23477 B<smiles>CC(=O)N(O)CCCC1C(=O)NC(CCCNC(=O)C(C)CCCO)C(=O)N1[18O]</smiles>

NBRI16716 A: $\mathrm{R}_{1}=\mathrm{OH}, \mathrm{R}_{2}=\mathrm{OH}$ NBRI16716 B: $R_{1}=H, R_{2}=\mathrm{OH}$

NBRI16716 C: $\mathrm{R}_{1}=\mathrm{OH}, \mathrm{R}_{2}=\mathrm{H}$

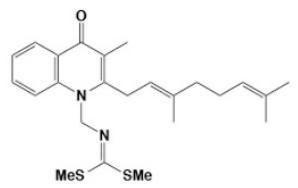

Intervenolin

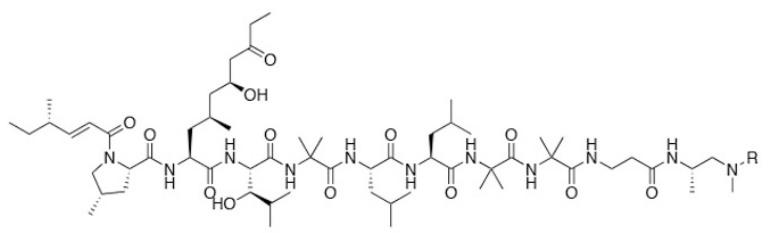

\section{Leucinostatin A: $\mathbf{R}=\mathrm{CH}_{3}$ Leucinostatin $\mathrm{B}: \mathbf{R}=\mathbf{H}$}

Figure 2 Modulators of tumor-stromal cell interactions. NBRI23477s, NBRI16716s and intervenolin are novel compounds discovered in our laboratory.

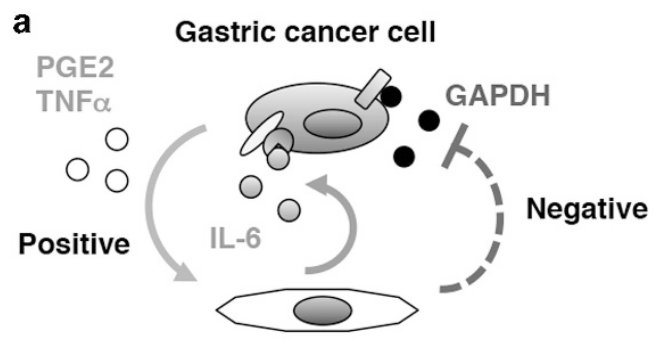

Gastric stromal cell

b

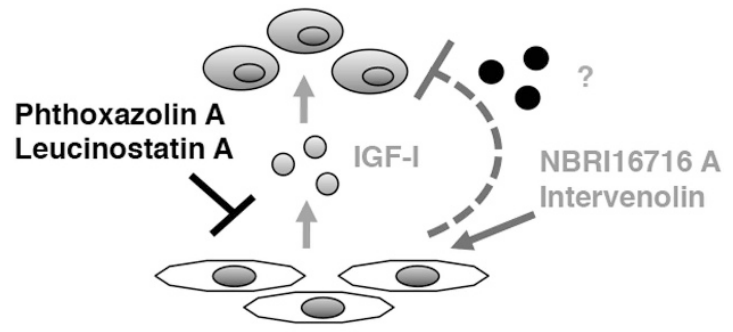

Stromal cells

Figure 3 Modulations of tumor-stromal cell interactions by small molecules. (a) Stromal cell interactions with gastric cancer cells include positive regulation by $\mathrm{TNF} \alpha$, PGE 2 and IL- 6 and also negative regulation by GAPDH. (b) Phthoxazolin A and leucinostatin A inhibit the secretion of IGF-I from stromal cells and suppress the growth of cancer cells. On the other hand, NBRI16716 A and intervenolin might stimulate stromal cells to secrete growth-inhibitory factors against cancer cells.

addition to gastric stromal cells and the secreted amounts are higher than cancer cells. ${ }^{5}$ The precise mechanism of secretion of GAPDH is still unknown, but we found that GAPDH does not need its endogenous dehydrogenase activity for the growth-inhibitory activity against cancer cells. ${ }^{5}$

\section{DISCOVERY OF ACTIVE COMPOUNDS FROM CULTURED BROTH OF MICROORGANISMS}

Prof. S Ōmura shared the 2015 Nobel Prize in Physiology or Medicine with Prof. W Cambell and Prof. Y Tu and his work has emphasized the utility of searching for useful compounds in cultured broths of microorganisms such as actinomycetes and fungi. Compounds isolated from such natural sources have unique structures and give us useful information especially in drug-development research. Similarly, we have been searching for active compounds from microorganisms' cultured broths using the above-mentioned co-culture system. As a result, we have discovered the following small active compounds.

Phthoxazolins and leucinostatins

Using a co-culture system of human prostate cancer and stromal cells we found several fungus cultured broth containing compounds that inhibited the growth of cancer cells in co-culture with stromal cells more strongly than that of cancer cells cultured alone. By isolation and structure determination those compounds were identified as phthoxazolin A and inthomycin B (a phthoxazolin derivative) from one fungus cultured broth ${ }^{7}$ and leucinostatin $\mathrm{A}$ and $\mathrm{B}$ from another one $^{8}$ (Figure 2). Although there are reports about phthoxazolins as an inhibitor of cellulose synthesis and leucinostatins as an antifungal compound, we have then found new activities. The mechanistic study revealed that phthoxazolin A and leucinostatin A were found to inhibit the production of IGF-I from prostate stromal cells resulting in suppression of prostate cancer cell growth in co-culture with the stromal cells (Figure 3b)., ${ }^{7,8}$ Furthermore, leucinostatins showed anti-tumor activity in vivo when prostate cancer cells were co-injected with stromal cells subcutaneously in nude mice. ${ }^{8}$ 


\section{NBRI23477s and NBRI16716s}

In a co-culture system of human prostate cancer cells and stromal cells, we discovered new compounds such as NBRI23477s, new atpenin derivatives, from the fungal strain Penicillium atramentosum PF1420 $0^{9}$ and NBRI16716s from a fungal strain Perisporiopsis melioloides Mer-f16716 ${ }^{10}$ (Figure 2). Since NBRI16716 C lacks the activity, a hydroxyl moiety linked to nitrogen is thought to be important for the activity of NBRI16716s. ${ }^{10}$ NBRI16716s did not show any adverse effects even at $50 \mathrm{mg} \mathrm{kg}^{-1}$ when administered to mice. As a result, NBRI16716 A and B exerted anti-tumor activity against xenograft models in vivo when human prostate cancer cells were co-injected subcutaneously with stromal cells into nude mice. ${ }^{10}$ Our preliminary experiments suggested that NBRI16716s did not affect IGF-I secretion from stromal cells, but it might stimulate the stromal cells to secrete some growth-inhibitory factors against cancer cells (Figure 3b).

\section{Intervenolins}

By co-culturing human gastric cancer and stromal cells, we discovered a novel compound, intervenolin, from the cultured broth of Nocardia sp. ML96-86F2 ${ }^{11}$ (Figure 2). We named it intervenolin, because it is a quinoline group compound intervening in tumor-stromal cell interactions. We obtained intervenolin at low concentrations (about $4 \mathrm{mg}$ per $10 \mathrm{l}$ culture broth). However, we successfully synthesized it ${ }^{12}$ as well as various derivatives. ${ }^{13}$ Intervenolin did not show any adverse effects on mice up to $50 \mathrm{mg} \mathrm{kg}^{-1}$ and it exerted potent anti-tumor effects against mouse xenograft models of human gastric and colorectal cancers. $^{11}$ Our preliminary results suggested that intervenolin might stimulate stromal cells to secrete growthinhibitory factors against cancer cells (Figure 3b). Furthermore, since compounds similar to intervenolin are reported to possess antiHelicobacter pylori activity, ${ }^{14}$ we assessed whether intervenolin also possessed the activity. We found that intervenolin (and its derivatives) showed potent and selective anti- $H$. pylori activity without any effect on intestinal bacteria. ${ }^{11,13}$ Thus, intervenolins are unique compounds possessing both anti-tumor and anti- $H$. pylori activities.

\section{PERSPECTIVE}

Other groups have reported the modulation of tumor-stromal cell interactions by small molecules. Hori et al. showed that naftopidil, a selective antagonist of the $\alpha 1$-adrenoceptor, inhibited IL- 6 secretion from prostate stromal cells and suppressed the growth of prostate cancer cells. ${ }^{15}$ Similarly, we hope that many researchers participate in this field and promote anti-tumor strategies targeting tumor-stromal cell interactions.

\section{CONFLICT OF INTEREST}

The authors declare no conflict of interest.

\section{ACKNOWLEDGEMENTS}

On winning the Sumiki-Umezawa Memorial Award 2015 from Japan Antibiotics Research Association, I thank all my colleagues and mentors, many of whom I cannot list in this limited space. However, I extend special thanks to Prof. M Shibasaki, Dr T Watanabe and H Abe for chemical synthetic studies, Dr M Igarashi, Dr M Hatano and C Hayashi for microbiological studies, $\mathrm{H}$ Inoue and S Ohba for animal experiments and Dr J Yoshida for mechanistic study.

1 Liao, C. P., Adisetiyo, H., Liang, M. \& Roy-Burman, P. Cancer-associated fibroblasts enhance the gland-forming capability of prostate cancer stem cells. Cancer Res. 70, 7294-7303 (2010)

2 Malanchi, I. et al. Interactions between cancer stem cells and their niche govern metastatic colonization. Nature 481, 85-89 (2012).

3 Kawada, M., Sakamoto, S. \& Nomoto, A. Modulation of tumor-stromal cell interactions: a new anti-tumor strategy targeting the tumor microenvironment. For. Immunopathol. Dis. Therap. 4, 53-62 (2013).

4 Kawada, M., Inoue, H., Masuda, T. \& Ikeda, D. Insulin-like growth factor I secreted from prostate stromal cells mediates tumor-stromal cell interactions of prostate cancer. Cancer Res. 66, 4419-4425 (2006).

5 Kawada, M. et al. Stromal cells positively and negatively modulate the growth of cancer cells: stimulation via the PGE2-TNF alpha-IL-6 pathway and inhibition via secreted GAPDH-E-cadherin interaction. PLOS ONE 10, e0119415 (2015)

6 Yamaji, R. et al. Glyceraldehyde-3-phosphate dehydrogenase in the extracellular space inhibits cell spreading. Biochim. Biophys. Acta 1726, 261-271 (2005).

7 Kawada, M., Inoue, H., Usami, I. \& Ikeda, D. Phthoxazolin A inhibits prostate cancer growth by modulating tumor-stromal cell interactions. Cancer Sci. 100, 150-157 (2009).

8 Kawada, M. et al. Leucinostatin A inhibits prostate cancer growth through reduction of insulin-like growth factor-I expression in prostate stromal cells. Int. J. Cancer 126, 810-818 (2010).

9 Kawada, M., Momose, I., Someno, T., Tsujiuchi, G. \& Ikeda, D. New atpenins, NBRI23477 A and B, inhibit the growth of human prostate cancer cells. J. Antibiot. (Tokyo) 62, 243-246 (2009).

10 Kawada, M. et al. NBRI16716A, a new antitumor compound against human prostate cancer cells, produced by Perisporiopsis melioloides Mer-f16716. J. Antibiot. (Tokyo) 63, 319-323 (2010)

11 Kawada, M. et al. Intervenolin, a new antitumor compound with anti-Helicobacter pylori activity, from Nocardia sp ML96-86F2. J. Antibiot. (Tokyo) 66, 543-548 (2013).

$12 \mathrm{Abe}, \mathrm{H}$. et al. Synthesis of intervenolin, an antitumor natural quino lone with unusual substituents. Org. Lett. 15, 2124-2127 (2013).

$13 \mathrm{Abe}, \mathrm{H}$. et al. Structure-activity relationship study of intervenolin derivatives: synthesis, antitumor, and anti-Helicobacter pylori activities. Tetrahedron 69, 7608-7617 (2013)

14 Dekker, K. A. et al. New quinolone compounds from Pseudonocardia sp. with selective and potent anti-Helicobacter pylori activity: taxonomy of producing strain, fermentation, isolation, structural elucidation and biological activities. J. Antibiot. (Tokyo) 51, 145-152 (1998).

15 Hori, Y. et al. Naftopidil, a selective \{alpha\}1-adrenoceptor antagonist, suppresses human prostate tumor growth by altering interactions between tumor cells and stroma. Cancer Prev. Res. (Phila.) 4, 87-96 (2011). 